\title{
Study on the application of interpolation in electromagnetic scattering characteristics
}

\author{
Liang Man ${ }^{\mathrm{a}}$, Haochuan Deng and Yanjie Cui \\ Science and Technology on Electromagnetic Scattering Laboratory, 100854 Beijing, China
}

\begin{abstract}
To get target ultra wideband electromagnetic scattering characteristics with high efficiency has great significance for the research of high resolution radar, imaging and target recognition. However accurately simulate the scattering characteristics of electrically large complex targets, the computational efficiency is very low. This paper study on the application of interpolation in the electromagnetic scattering characteristics, and use a typical warhead as example quantitative application range is presented.
\end{abstract}

Keywords: interpolation; RCS; scattering characteristics; simulation.

\section{Introduction}

Radar mainly work at microwave frequencies, common military targets such as missile and aircraft are electrically large complex targets, how to get target ultra wideband electromagnetic scattering characteristics with high efficiency, has great significance for the research of high resolution radar, imaging and target recognition. However ultra-wideband radar work frequency band is very wide, using the traditional numerical method to accurately analysis [1] the scattering characteristics of each frequency point in the frequency band, electromagnetic scattering must be calculated point by point, and computational efficiency is very low. Therefore, how to use the electromagnetic information carried by a few frequency point of the frequency band to get the electromagnetic scattering characteristics of the whole frequency band is particularly important, introducing the interpolation method to solve the above problem.

\section{The interpolation definition and method}

The raise of interpolation problem: in actual production and science, the function relationship between the variables is studied, but in a lot of cases it is hard to find a specific function, and can only acquire a function table through measurement or observation, this function express by a form will not be able to work out the function values not in the table, so the properties of the function can't be further studied. Some may give a function expression, but the formula is very complex, and is not suitable for use. In order to solve these problems, we hope to calculate a simple function $\mathrm{P}(\mathrm{x})$ according to the given function table, the method called interpolation method.

Definition: suppose function $y=f(x)$ is defined on the interval [a, b], and the value on the

a Corresponding author : manliang89@163.com

(C) 2016. The authors - Published by Atlantis Press 
point $a \leq x_{0}<x_{1}<\cdots<x_{n} \leq b$ are $y_{0}, y_{1}, \cdots, y_{n}$, if there is a simple function $\mathrm{P}(\mathrm{x})$, make

$$
P\left(x_{i}\right)=y_{i}, \quad i=0,1, \cdots, n
$$

Come into existence, it is said $\mathrm{P}(\mathrm{x})$ is the interpolation function of $\mathrm{f}(\mathrm{x})$, points $a \leq x_{0}<x_{1}<\cdots<x_{n} \leq b$ are called interpolation node, the in interval [a, b] is called interpolation interval, the method to calculate $\mathrm{P}(\mathrm{x})$ is called interpolation method. The commonly used interpolation method, Lagrange interpolation, Hermite interpolation, Newton interpolation, etc [2, 3]. A specific interpolation method is given below.

Given the value $(y=f(x))$ of $N$ number not equidistant points $x(i)$, use Lagrange interpolation formula to calculate the interpolation points $t$ approximation value $\mathrm{z}=\mathrm{f}(\mathrm{t})$.

In order to avoid the runge phenomenon affect on the result of calculation, eight points are arbitrary selected from the $\mathrm{N}$ points, and the interpolation point $\mathrm{t}$ in among them, mean chose:

$\mathrm{x}_{\mathrm{k}}<\mathrm{x}_{\mathrm{k}+1}<\mathrm{x}_{\mathrm{k}+2}<\mathrm{x}_{\mathrm{k}+3}<\mathrm{t}<\mathrm{x}_{\mathrm{k}+4}<\mathrm{x}_{\mathrm{k}+5}<\mathrm{x}_{\mathrm{k}+6}<\mathrm{x}_{\mathrm{k}+7}$

Lagrange interpolation polynomial is used to calculate approximation value $\mathrm{z}=\mathrm{f}$ (t) of the interpolation points,

$$
Z=\sum_{i=k}^{k+7} y_{i} \coprod_{\substack{j=k \\ j \neq i}}^{k+7}\left[\left(t-x_{j}\right) /\left(x_{i}-x_{j}\right)\right]
$$

When interpolation point $t$ locates outside the interval which includes $n$ points, just take four point of one end of the interval to interpolate, and when the interpolation point $t$ near the ends of the n nodes, the selected points is less than eight.

\section{Typical warhead interpolation analysis}

Select the cone warhead, the shape is shown as figure1. $\theta$ is pitch angle. $\varphi$ is azimuth. Respectively, $\mathrm{X}$ band and $\mathrm{C}$ band data are simulated. Simulation conditions: $\mathrm{X}$ band, frequency $10 \mathrm{GHz}, \mathrm{C}$ band, frequency 5.6GHz; Pitch angle 90 degrees; Azimuth 0 180 degrees, angle-interval 0.01 degrees. In this paper, the azimuth angle is interpolated, and error of the interpolation is set at $0.2 \mathrm{~dB}, 0.02$ degrees data, 0.05 degrees data, 0.1 degrees data and 0.2 degrees data (namely two times interpolation, five times interpolation, ten times interpolation, twenty times interpolation) are used to interpolate 0.01 degrees original data respectively, and calculate error of the interpolation, analysis the feasibility of the interpolation.

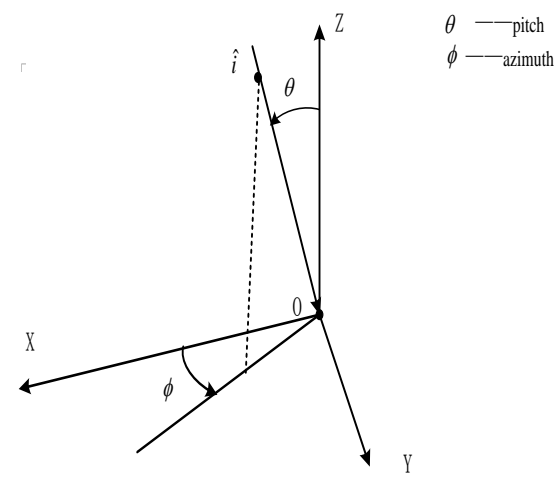

Figure 1. Target position 


\subsection{The X-band interpolation analysis}

Figure $2 \sim$ Figure 5 are results of two times interpolation, five times interpolation, ten times interpolation and twenty times interpolation of the warhead in $\mathrm{X}$ band $\mathrm{HH}$ polarization.

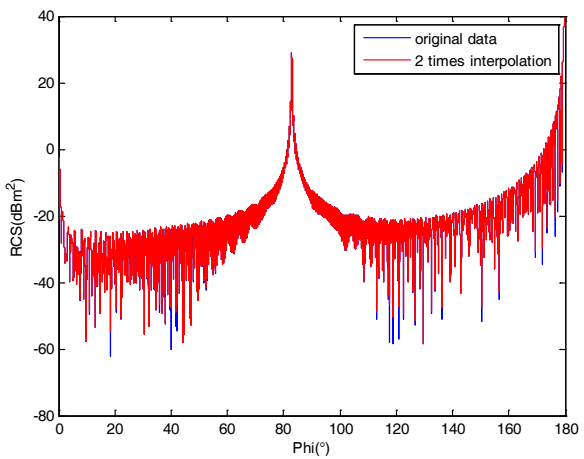

Figure 2. HH polarization 2 times interpolation

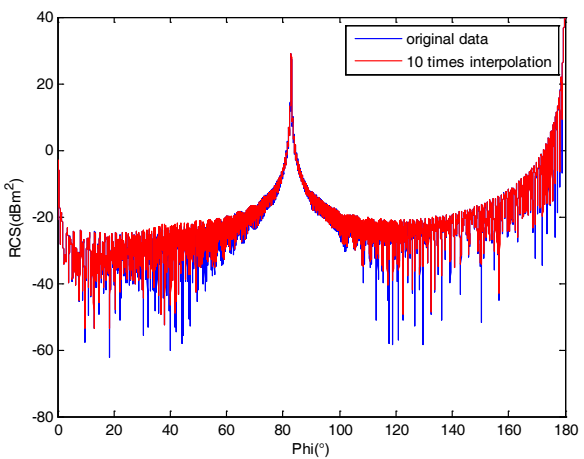

Figure 4. HH polarization 10 times interpolation

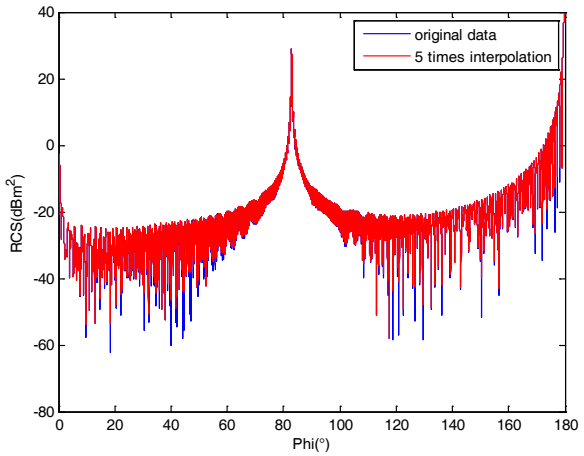

Figure 3. HH polarization 5 times interpolation

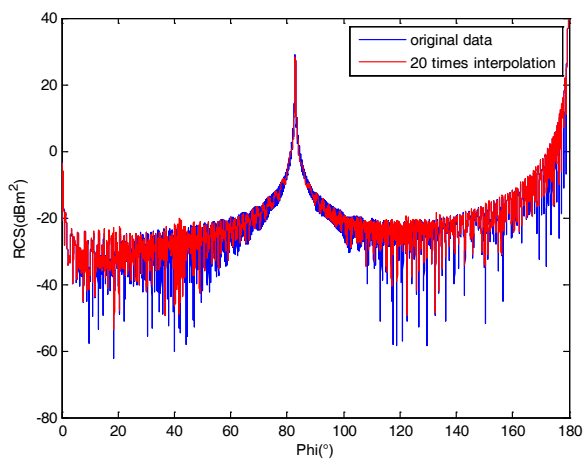

Figure 5. HH polarization 20 times interpolation

Among them, 2 times interpolation $\mathrm{HH}$ polarization average error for the interpolation results is $0.0212 \mathrm{~dB}$, the variance is 0.0683 ; 5 times interpolation $\mathrm{HH}$ polarization average error for the interpolation results is $0.1484 \mathrm{~dB}$, the variance is 0.4418 ; 10 times interpolation $\mathrm{HH}$ polarization average error for the interpolation results is $0.5790 \mathrm{~dB}$, the variance is 1.6282 ; 20 times interpolation $\mathrm{HH}$ polarization average error for the interpolation results is $1.7340 \mathrm{~dB}$, the variance is 5.5754 . Table 1 is segmentation error statistical results.

Table 1. HH polarization 10GHz error statistical

\begin{tabular}{|c|c|c|c|c|}
\hline $\begin{array}{c}\text { azimuth }\left({ }^{\circ}\right) \\
\text { Interpolation }\end{array}$ & $0 \sim 180$ & $0 \sim 60$ & $60 \sim 100$ & $100 \sim 180$ \\
\hline 2 times & 0.0212 & 0.0370 & $8.3 \mathrm{e}-4$ & 0.0194 \\
\hline 5 times & 0.1484 & 0.2444 & 0.0488 & 0.1262 \\
\hline 10 times & 0.5490 & 0.8402 & 0.4652 & 0.4399 \\
\hline 20 times & 1.7340 & 2.5035 & 1.3168 & 1.3649 \\
\hline
\end{tabular}




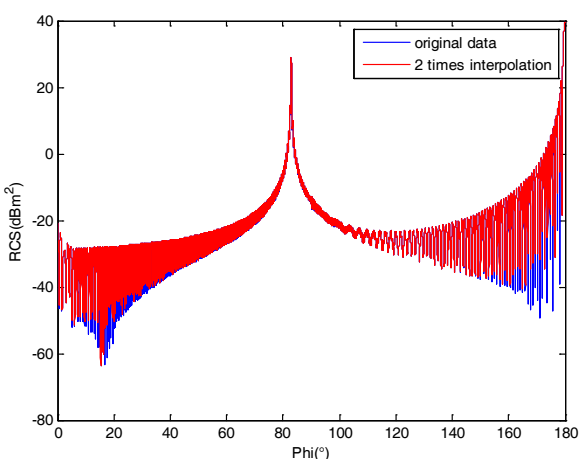

Figure 6. VV polarization 2 times interpolation

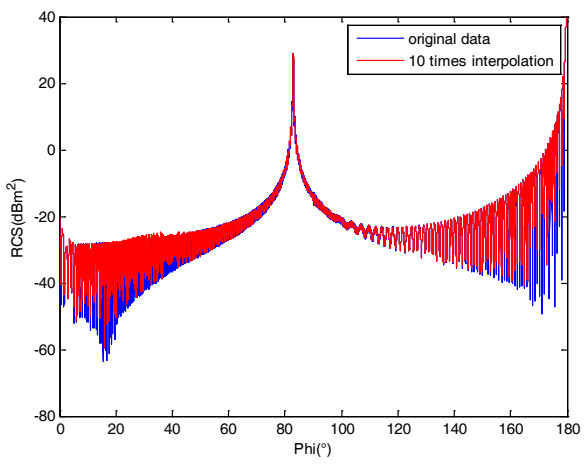

Figure 8. VV polarization 10 times interpolation

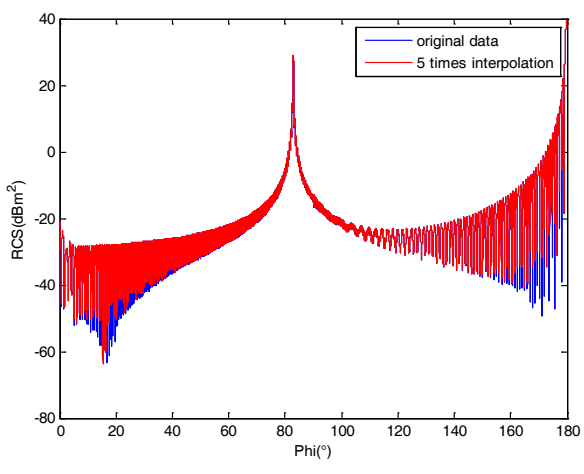

Figure 7. VV polarization 5 times interpolation

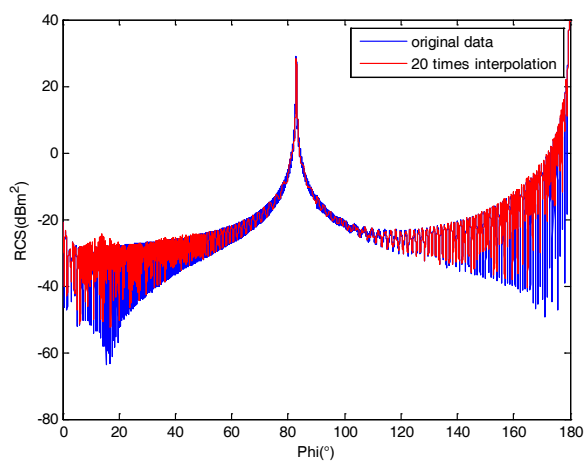

Figure 9. VV polarization 20 times interpolation

Figure $6 \sim$ Figure 9 are results of two times interpolation, five times interpolation, ten times interpolation and twenty times interpolation of the warhead in $\mathrm{X}$ band VV polarization.

Among them, 2 times interpolation VV polarization average error for the interpolation results is $0.0251 \mathrm{~dB}$, the variance is 0.1490 ; 5 times interpolation VV polarization average error for the interpolation results is $0.1655 \mathrm{~dB}$, the variance is $0.6693 ; 10$ times interpolation $\mathrm{VV}$ polarization average error for the interpolation results is $0.6042 \mathrm{~dB}$, the variance is $1.8487 ; 20$ times interpolation VV polarization average error for the interpolation results is $1.7639 \mathrm{~dB}$, the variance is 5.9153 . Table 2 is segmentation error statistical results.

Table 2. VV polarization $10 \mathrm{GHz}$ error statistical

\begin{tabular}{|c|c|c|c|c|}
\hline Interpolation $^{\text {azimuth }}{ }^{\circ}$ & $0 \sim 180$ & $0 \sim 60$ & $60 \sim 100$ & $100 \sim 180$ \\
\hline 2 times & 0.0251 & 0.0213 & $3.15 \mathrm{e}-4$ & 0.0699 \\
\hline 5 times & 0.1655 & 0.2241 & 0.0129 & 0.2768 \\
\hline 10 times & 0.6042 & 0.8673 & 0.1911 & 0.6973 \\
\hline 20 times & 1.7639 & 2.5598 & 0.7426 & 1.7032 \\
\hline
\end{tabular}

It can be observed from the table that $\mathrm{HH}$ polarization 2 times and 5 times interpolation can satisfy RCS error requirement, in which 5 times interpolation 0 60 degrees is not satisfy; VV polarization 2 times interpolation can satisfy RCS error requirement, and 10 times interpolation 60 100 degrees is also satisfy RCS error requirement. That is, In X band 2 times interpolation can be used to reduction all aspect RCS value, HH polarization 60 180 degrees data can use 5 times interpolation to reduction, 
VV polarization 60 100 degrees data can use 10 times interpolation to reduction.

\subsection{The C-band interpolation analysis}

Figure $10 \sim$ Figure 13 are results of two times interpolation, five times interpolation, ten times interpolation and twenty times interpolation of the warhead in C-band $\mathrm{HH}$ polarization.

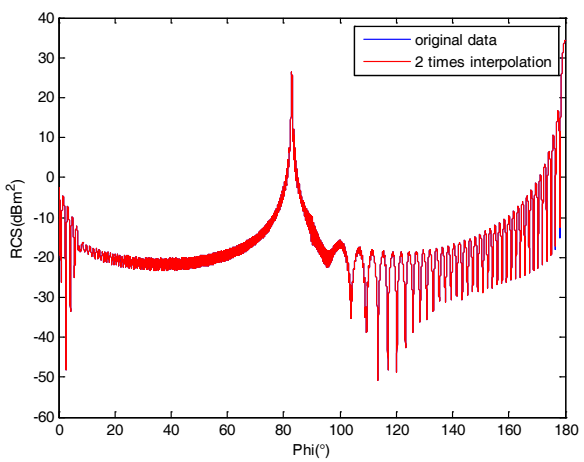

Figure 10. HH polarization 2 times interpolation

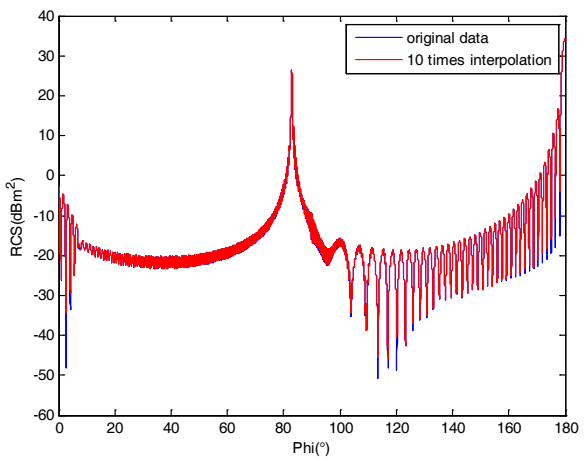

Figure 12. HH polarization 10 times interpolation

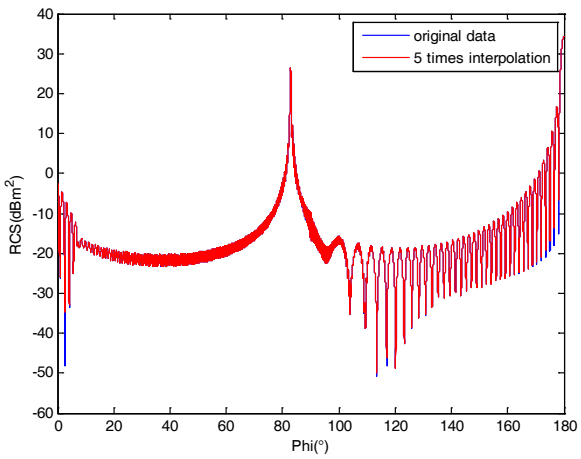

Figure 11. HH polarization 5 times interpolation

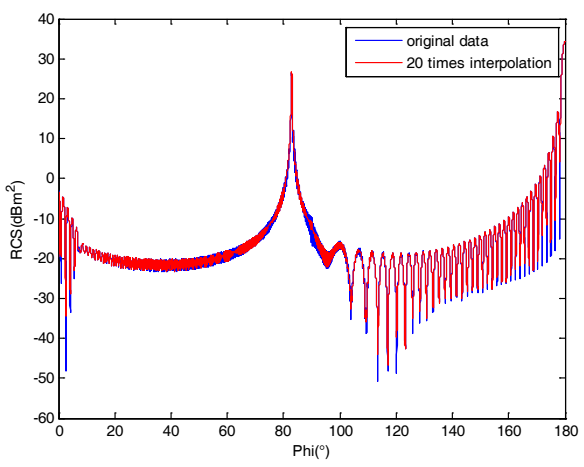

Figure 13. HH polarization 20 times interpolation

Table 3. HH polarization 5.6GHz error statistical

\begin{tabular}{|c|c|c|c|c|}
\hline Interpolation & $0 \sim 180$ & $10 \sim 60$ & $60 \sim 130$ & $130 \sim 160$ \\
\hline 2 times & 0.0019 & $6.856 \mathrm{e}-4$ & $5.453 \mathrm{e}-4$ & $4.055 \mathrm{e}-4$ \\
\hline 5 times & 0.0134 & 0.0017 & 0.0079 & 0.0012 \\
\hline 10 times & 0.0688 & 0.0138 & 0.0829 & 0.0174 \\
\hline 20 times & 0.4105 & 0.1708 & 0.6004 & 0.1613 \\
\hline
\end{tabular}

Among them, 2 times interpolation $\mathrm{HH}$ polarization average error for the interpolation results is $0.0019 \mathrm{~dB}$, the variance is 0.0030 ; 5 times interpolation $\mathrm{HH}$ polarization average error for the interpolation results is $0.0134 \mathrm{~dB}$, the variance is 0.0385 ; 10 times interpolation $\mathrm{HH}$ polarization average error for the interpolation results is $0.0688 \mathrm{~dB}$, the variance is $0.1371 ; 20$ times interpolation $\mathrm{HH}$ polarization average error for the interpolation results is $0.4105 \mathrm{~dB}$, the variance is 0.6354 . Table 3 is segmentation error statistical results. 


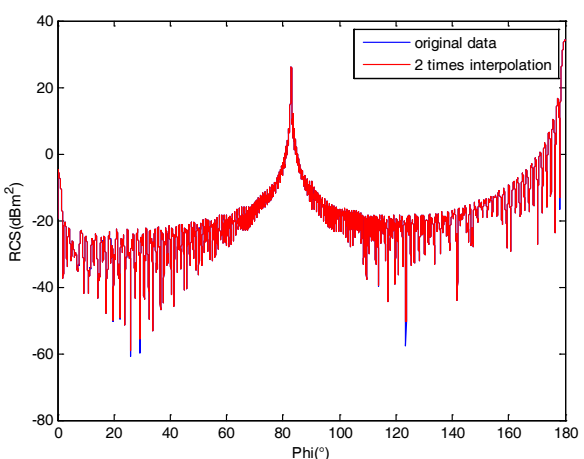

Figure 14. VV polarization 2 times interpolation

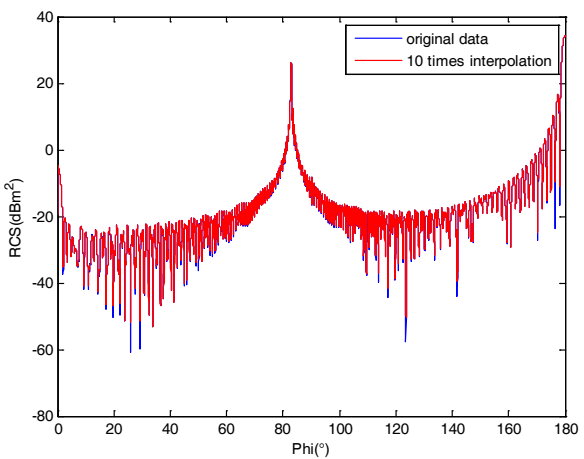

Figure 16. VV polarization 10 times interpolation

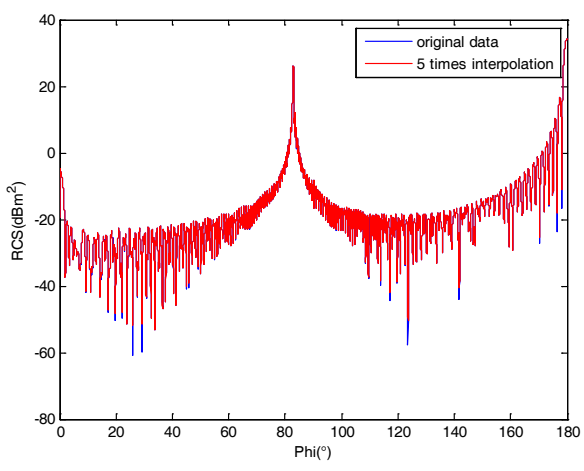

Figure 15. VV polarization 5 times interpolation

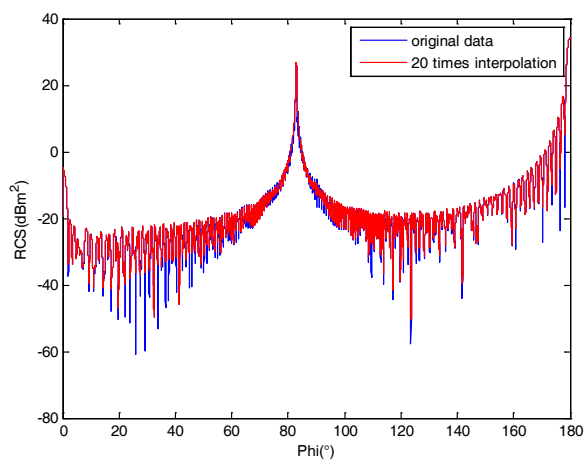

Figure 17. VV polarization 20 times interpolation

Figure $14 \sim$ Figure 17 are results of two times interpolation, five times interpolation, ten times interpolation and twenty times interpolation of the warhead in $\mathrm{C}$ band VV polarization.

Among them, 2 times interpolation VV polarization average error for the interpolation results is $0.0054 \mathrm{~dB}$, the variance is 0.0087 ; 5 times interpolation $\mathrm{VV}$ polarization average error for the interpolation results is $0.0311 \mathrm{~dB}$, the variance is 0.0632 ; 10 times interpolation $\mathrm{VV}$ polarization average error for the interpolation results is $0.1413 \mathrm{~dB}$, the variance is $0.2857 ; 20$ times interpolation VV polarization average error for the interpolation results is $0.6219 \mathrm{~dB}$, the variance is 1.5919 . Table 4 is segmentation error statistical results.

Table 4. VV polarization 5.6GHz error statistical

\begin{tabular}{|c|c|c|c|c|}
\hline azimuth $\left(^{\circ}\right)$ & $0 \sim 180$ & $10 \sim 60$ & $60 \sim 130$ & $130 \sim 160$ \\
\hline 2 times & 0.0054 & 0.0106 & $7.765 \mathrm{e}-4$ & $6.899 \mathrm{e}-4$ \\
\hline 5 times & 0.0311 & 0.0624 & 0.0081 & 0.0065 \\
\hline 10 times & 0.1413 & 0.2541 & 0.1091 & 0.0406 \\
\hline 20 times & 0.6219 & 0.8659 & 0.7003 & 0.2348 \\
\hline
\end{tabular}

It can be observed from the table that HH polarization 10 times interpolation can satisfy RCS error requirement, and 20 times interpolation 10 60 degrees and 130 160 degrees is also satisfy RCS error requirement.; VV polarization 5 times interpolation can satisfy RCS error requirement, and 10 times interpolation 60 160 degrees is also satisfy RCS error requirement. That is, In C band 5 times interpolation can be used to reduction all aspect RCS value. in which , HH polarization 10 times 
interpolation can be used to reduction all aspect RCS value , 10 60 degrees and 130 160 degrees data can use 20 times interpolation to reduction; VV polarization 60 160 degrees data can use 10 times interpolation to reduction.

\section{Conclusion}

Simulation results show that, within a certain range of error, the interpolation data can reflect the features of raw data well. Therefore, when to calculate the electromagnetic scattering characteristics of electrically large target, we can use less data by interpolation to reach a certain high resolution, that greatly improve the efficiency of simulation

\section{References}

1. Huang Peikang, Yin Hongcheng, Xu Xiaojian. Radar Target Characteristic(Publishing House of Electronics Industry, 2005)

2. Jiang Yaolin. Modern Numerical Analysis( National Defense Industry Press,2004)

3. Zhu Changqing. Numerical calculation method and its application(Science Publishing Company, 2006) 